\title{
Endoscopic Criteria for Evaluating Tumor Stage after Preoperative Chemoradiation Therapy in Locally Advanced Rectal Cancer
}

\section{Kyung Su Han, MD \\ Dae Kyung Sohn, MD \\ Dae Yong Kim, MD \\ Byung Chang Kim, MD \\ Chang Won Hong, MD \\ Hee Jin Chang, MD \\ Sun Young Kim, MD \\ Ji Yeon Baek, MD \\ Sung Chan Park, MD \\ Min Ju Kim, MD \\ Jae Hwan Oh, MD}

Center for Colorectal Cancer,

Research Institute and Hospital,

National Cancer Center, Goyang, Korea

\begin{abstract}
Purpose
Local excision may be an another option for selected patients with markedly down-staged rectal cancer after preoperative chemoradiation therapy (CRT), and proper evaluation of post-CRT tumor stage (ypT) is essential prior to local excision of these tumors. This study was designed to determine the correlations between endoscopic findings and ypT of rectal cancer.
\end{abstract}

\section{Materials and Methods}

In this study, 481 patients with locally advanced rectal cancer who underwent preoperative CRT followed by surgical resection between 2004 and 2013 at a single institution were evaluated retrospectively. Pathological good response ( $p-G R)$ was defined as ypT $\leq 1$, and pathological minimal or no response $(\mathrm{p}-\mathrm{MR})$ as $\mathrm{ypT} \geq 2$. The patients were randomly classified according to two groups, a testing $(n=193)$ and a validation $(n=288)$ group. Endoscopic criteria were determined from endoscopic findings and ypT in the testing group and used in classifying patients in the validation group as achieving or not achieving $\mathrm{p}-\mathrm{GR}$.

\section{Results}

Based on findings in the testing group, the endoscopic criteria for p-GR included scarring, telangiectasia, and erythema, whereas criteria for $\mathrm{p}-\mathrm{MR}$ included nodules, ulcers, strictures, and remnant tumors. In the validation group, the kappa statistic was 0.965 ( $p<0.001$ ), and the sensitivity, specificity, positive predictive value, and negative predictive value were $0.362,0.963,0.654$, and 0.885 , respectively.

\section{Conclusion}

The endoscopic criteria presented are easily applicable for evaluation of ypT after preoperative CRT for rectal cancer. These criteria may be used for selection of patients for local excision of down-staged rectal tumors, because patients with $\mathrm{p}-\mathrm{MR}$ could be easily ruled out.

\author{
Correspondence: Dae Kyung Sohn, MD \\ Center for Colorectal Cancer, \\ Research Institute and Hospital, \\ National Cancer Center, 323 Ilsan-ro, \\ Ilsandong-gu, Goyang 10408, Korea \\ Tel: 82-31-920-1636 \\ Fax: 82-31-920-1289 \\ E-mail: gsgsbal@ncc.re.kr \\ Received June 3, 2015 \\ Accepted September 1, 2015 \\ Published Online September 22, 2015
}

\section{Key words}

Rectal neoplasms, Chemoradiotherapy, Neoadjuvant therapy, Response Evaluation Criteria in Solid Tumors, Endoscopy

\section{Introduction}

Preoperative chemoradiation therapy (CRT) is widely accepted as an effective treatment for patients with locally advanced low rectal cancer [1]. Of patients who undergo preoperative CRT followed by radical surgery, 10\%-30\% achieve complete regression (CR), defined as the histopathologically confirmed absence of residual tumor cells [2]. Although radical resection is still the standard treatment for rectal cancer, local excision has been accepted as an alternative for patients who achieve CR or selective good response after preoperative CRT [3-10].

A recent phase II clinical trial of local excision after preop- 
erative CRT for rectal cancer suggested that the local excision may be a good option for selected patients with rectal cancer if post-CRT tumor stage (ypT) is "0" or "1" [11]. However, prior to local excision, proper evaluation of $\mathrm{ypT}$ is essential.

Endoscopy is known to be more effective than other methods for evaluation of ypT, and several recent studies have reported the endoscopic findings associated with clinical CR or good response after preoperative CRT for distal rectal cancer [12-14]. However, those studies included a relatively small number of patients, and their proposed endoscopic findings were not standardized. The current study was therefore designed to determine the correlations between endoscopic findings and ypT of rectal cancer, and to suggest endoscopic criteria for evaluation of ypT of rectal cancer.

\section{Materials and Methods}

Between 2004 and 2013, a total of 520 patients with histologically confirmed rectal cancer with or without lymph node metastasis underwent surgery after preoperative CRT at the National Cancer Center, Korea. In these patients the tumors were located in the mid or distal rectum, clinically diagnosed as CT3 or CT4, and showed no distant metastasis.

After exclusion of 39 patients, due to intervals between the end of preoperative CRT and surgery of $<40$ days or $>65$ days, 481 patients were included in this study. This study was approved by the Institutional Review Board of the National Cancer Center, Korea (NCC2014-0005).

According to the standard treatment protocol of our institution, all patients received 45 Gy pelvic radiation therapy in 25 fractions followed by a $5.4 \mathrm{~Gy}$ boost in three fractions within 6 weeks, and preoperative chemotherapy was started on the first day of pelvic radiotherapy and delivered concurrently with radiotherapy. All patients received a fluoropyrimidine-based regimen, consisting of (1) 5-fluorouracil and leucovorin, (2) capecitabine, (3) capecitabine and irinotecan, or (4) oral tegafur and uracil. Tumor resections were performed 6-8 weeks after the last dose of CRT. All patients underwent two endoscopic evaluations, shortly before preoperative CRT and just before surgery.

Clinical and pathological data were reviewed and endoscopic images were collected from our database. Pathological response was determined by histopathologic examination of tumor specimens. A pathological good response ( $\mathrm{p}-\mathrm{GR}$ ) was defined as ypT0, ypTis, or ypT1, whereas a pathological minimal or no response (p-MR) was defined as ypT2-4.

The 481 enrolled patients were randomized into two patient cohorts using a computer generated random sampling, with 193 included in the testing set and 288 included in the validation set. Three expert endoscopists (A, B, and C) evaluated the endoscopic findings based on recorded endoscopic pictures, and determined by consensus the standard endoscopic findings after preoperative CRT based on previous reports [12-14]. These findings included scarring, telangiectasia, erythema, nodules, ulcers, strictures, and remnant tumor, as defined in Table 1.

\section{Testing set}

Endoscopist A performed evaluation of the testing set. Preoperative endoscopic findings and postoperative histopathologic results of 193 randomly selected patients were reviewed. Following analysis of the endoscopic characteristics used to differentiate between $\mathrm{p}-\mathrm{GR}$ and $\mathrm{p}-\mathrm{MR}$, the patients were classified according to two groups: those with endoscopic findings for p-GR (e-GR) and p-MR (e-MR).

\section{Validation set}

Endoscopists B and C evaluated the validation set. These two endoscopists reviewed and classified endoscopic findings of 288 patients without information on their ypT classification, using the endoscopic criteria determined in the testing group. Agreement between e-GR and p-GR was analyzed.

\section{Statistical analyses}

Continuous variables were compared using Student's $t$ test and categorical variables using the chi-squared or Fisher exact test, as appropriate. The validity of the endoscopic criteria was determined by calculating kappa value, sensitivity, specificity, positive predictive value (PPV), negative predictive value (NPV), and positive and negative likelihood ratios.

p-values of $<0.05$ were considered statistically significant. All statistical analyses were performed using SPSS ver. 14.0 (SPSS Inc., Chicago, IL).

\section{Results}

The 481 included patients consisted of 344 men and 137 women, with a mean age of 59.7 years (range, 27 to 87 years). Of these, 478 patients underwent radical resection and three patients underwent local excision. Mean tumor sizes were $5.5 \mathrm{~cm}$ (range, 1.5 to $12 \mathrm{~cm}$ ) before CRT and $2.7 \mathrm{~cm}$ (range, 0 to $9.5 \mathrm{~cm}$ ) after CRT. Of these patients, $53(11.0 \%)$ had ypT0 tumors, nine $(1.9 \%)$ had ypTis, $26(5.4 \%)$ had ypT1, 112 
Table 1. Definition of endoscopic findings for evaluating tumor response after preoperative chemoradiation

\begin{tabular}{ll} 
Finding & Definition \\
$\begin{array}{l}\text { Good response } \\
\text { Scarring }\end{array}$ & Flattening and whitening of the mucosa with fibrotic changes \\
Telangiectasia & Scarring surrounded by small blood vessels \\
\hline $\begin{array}{l}\text { Erythema } \\
\text { Minimal or no response }\end{array}$ & Scarring or erosion with peripheral erythematous mucosal changes \\
\hline Nodule & No definite tumor but a residual small mucosal lump \\
\hline Ulcer & Any residual ulceration with a necrotic or regenerative bed \\
\hline Stricture & Luminal narrowing with over $50 \%$ reduction in luminal diameter \\
\hline Remnant tumor & Definite residual tumor with or without ulceration \\
\hline
\end{tabular}

Table 2. Patient demographic and tumor characteristics

\begin{tabular}{|c|c|c|c|c|}
\hline Characteristic & Total $(n=481)$ & Testing ( $n=193)$ & Validation $(\mathrm{n}=288)$ & p-value \\
\hline Age (yr) & $59.7(27-87)$ & 59.5 & 59.8 & ns \\
\hline \multicolumn{5}{|l|}{ Sex } \\
\hline Male & 344 & 136 & 208 & ns \\
\hline Female & 137 & 57 & 80 & \\
\hline \multicolumn{5}{|l|}{ Histologic grade } \\
\hline Low & 461 & 182 & 279 & ns \\
\hline High & 20 & 11 & 9 & \\
\hline \multicolumn{5}{|l|}{ Tumor size $(\mathrm{cm})$} \\
\hline Pre-CRT & $5.5(1.5-12)$ & 5.7 & 5.6 & ns \\
\hline Post-CRT & $2.7(0-9.5)$ & 2.5 & 2.8 & ns \\
\hline Distance from anal verge $(\mathrm{cm})$ & $5.7(0-13)$ & 5.7 & 5.7 & ns \\
\hline \multicolumn{5}{|l|}{ Clinical T classification } \\
\hline cT3 & $450(93.6)$ & 182 & 268 & ns \\
\hline cT4 & $31(6.4)$ & 11 & 20 & \\
\hline \multicolumn{5}{|l|}{ Operation type } \\
\hline Low anterior resection & 435 & 175 & 260 & ns \\
\hline Abdominoperineal resection & 43 & 16 & 27 & \\
\hline Transanal excision & 3 & 2 & 1 & \\
\hline \multicolumn{5}{|l|}{ Pathologic T classification } \\
\hline урТ0 & $53(11.0)$ & 25 & 28 & ns \\
\hline ypTis & $9(1.9)$ & 4 & 5 & \\
\hline ypT1 & $26(5.4)$ & 12 & 14 & \\
\hline урТ2 & $112(23.3)$ & 39 & 73 & \\
\hline урT3 & $256(53.2)$ & 103 & 153 & \\
\hline ypT4 & $25(5.2)$ & 10 & 15 & \\
\hline \multicolumn{5}{|l|}{ Dworak's regression grade } \\
\hline 4 (total regression) & $48(10.0)$ & 23 & 25 & ns \\
\hline 3 (near-total regression) & $60(12.5)$ & 21 & 39 & \\
\hline 2 (moderate regression) & $270(56.1)$ & 112 & 158 & \\
\hline 1 (minimal regression) & 99 (20.6) & 36 & 63 & \\
\hline 0 (no regression) & $4(0.8)$ & 1 & 3 & \\
\hline
\end{tabular}

Values are presented as mean (range) or number (\%). ns, not significant; CRT, chemoradiation therapy. 
Table 3. Endoscopic findings according to pathologic response in the testing set

\begin{tabular}{|c|c|c|}
\hline Endoscopic finding & p-GR & p-MR \\
\hline \multicolumn{3}{|l|}{ e-GR findings } \\
\hline Scarring & $8(19.5)$ & $4(2.6)$ \\
\hline Erythema & $8(19.5)$ & $4(2.6)$ \\
\hline Telangiectasia & $5(12.2)$ & $2(1.4)$ \\
\hline \multicolumn{3}{|l|}{ e-MR findings } \\
\hline Nodules & $2(4.9)$ & $8(5.3)$ \\
\hline Ulcer & $13(31.7)$ & $64(42.1)$ \\
\hline Stricture & $4(9.8)$ & $28(18.4)$ \\
\hline Remnant tumor & $1(2.4)$ & $42(27.6)$ \\
\hline Total & 41 & 151 \\
\hline
\end{tabular}

Values are presented as number (\%). p-GR, pathological good response; $\mathrm{p}-\mathrm{MR}$, pathological minimal or no response; e-GR, endoscopic good response; e-MR, endoscopic minimal or no response.
Table 4. Endoscopic findings evaluated by two endoscopists in the validation set

\begin{tabular}{|lcc|}
\hline Endoscopic finding & Endoscopist B & Endoscopist C \\
\hline e-GR findings & & \\
\hline Scarring & $9(3.1)$ & $8(2.7)$ \\
\hline Erythema & $13(4.5)$ & $12(4.2)$ \\
\hline Telangiectasia & $4(1.4)$ & $4(1.4)$ \\
\hline e-MR findings & & \\
\hline Nodule & $17(5.9)$ & $8(2.8)$ \\
\hline Ulcer & $194(67.4)$ & $192(66.7)$ \\
\hline Stricture & $7(2.4)$ & $24(8.3)$ \\
\hline Remnant tumor & $44(15.3)$ & $40(13.9)$ \\
\hline Total & 288 & 288 \\
\hline
\end{tabular}

Values are presented as number (\%). e-GR, endoscopic good response; e-MR, endoscopic minimal or no response.
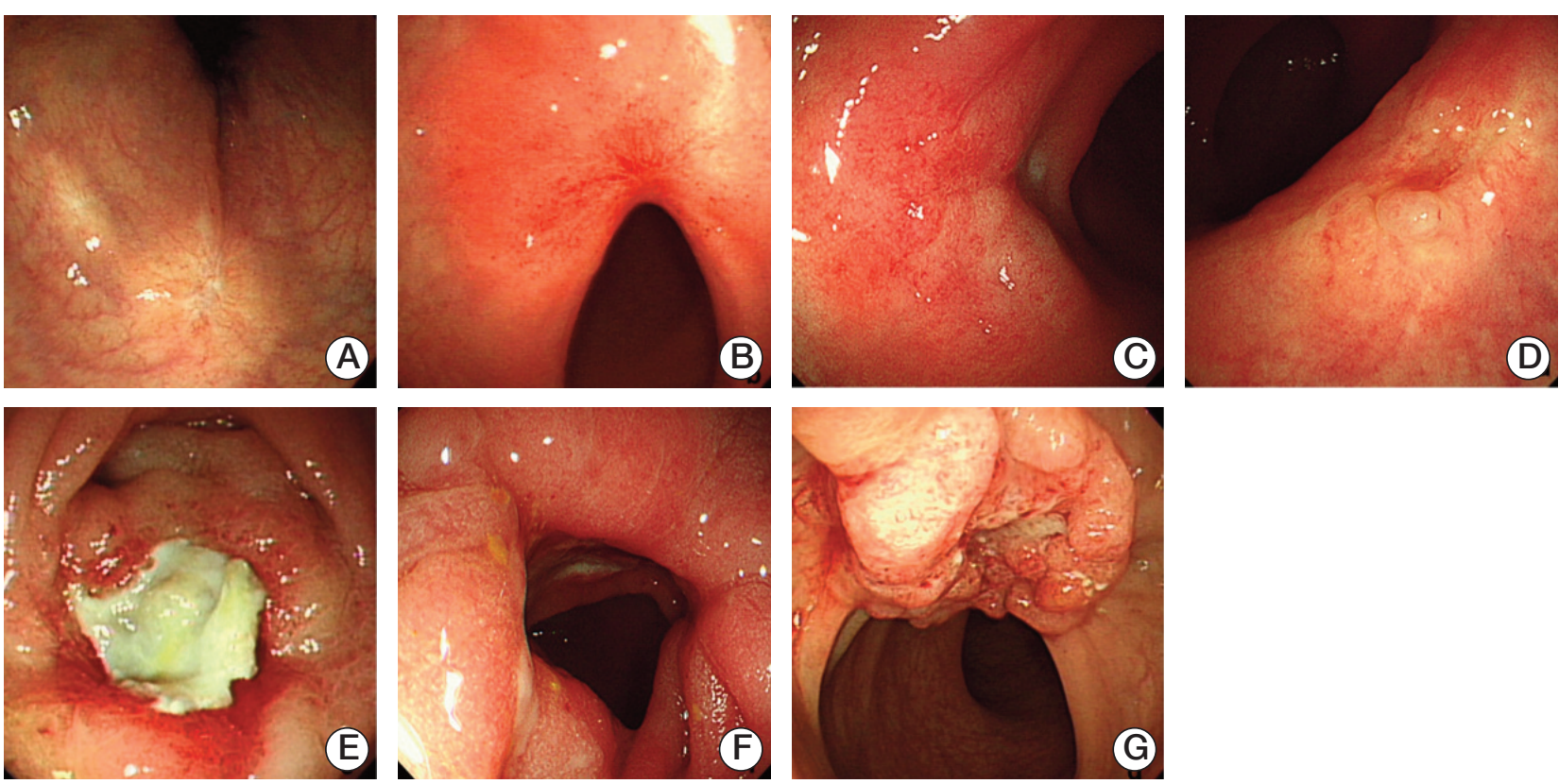

Fig. 1. Endoscopic findings for evaluation of tumor response after preoperative chemoradiation. (A) Scarring. (B) Telangiectasia. (C) Erythema. (D) Nodule. (E) Ulcer. (F) Stricture. (G) Remnant tumor.

(23.3\%) had ypT2, $256(53.2 \%)$ had ypT3, and $25(5.2 \%)$ had ypT4 (Table 2).

\section{Testing set}

Three endoscopic characteristics were associated with p-GR (e-GR): scarring, telangiectasia, and erythema (Table 3,
Fig. 1), while four endoscopic characteristics were associated with p-MR (e-MR): nodule, ulcer, stricture, and remnant tumor (Fig. 1). The sensitivity and specificity of these endoscopic characteristics in predicting p-GR were 0.538 and 0.935, respectively, whereas the PPV and NPV were 0.677 and 0.889 , respectively. 
Table 5. Validities of predicting pathological good response based on endoscopic findings

\begin{tabular}{lccc} 
& Testing set & \multicolumn{2}{c}{ Validation set } \\
\cline { 2 - 4 } Statistic & Endoscopist A & Endoscopist B & Endoscopist C \\
Sensitivity & 0.538 & 0.362 & 0.277 \\
Specificity & 0.935 & 0.963 & 0.959 \\
Positive predictive value & 0.677 & 0.654 & 0.565 \\
Negative predictive value & 0.889 & 0.885 & 0.872 \\
False positive ratio & 0.323 & 0.346 & 0.435 \\
False negative ratio & 0.111 & 0.115 & 0.128 \\
Positive likelihood ratio & 8.292 & 9.686 & 6.666 \\
Negative likelihood ratio & 0.494 & 0.663 & 0.755 \\
\hline
\end{tabular}

\section{Validation set}

Using the endoscopic criteria determined in the testing set, classification of the 288 patients in the validation set was performed by two endoscopists (Table 4). The kappa statistic for inter-observer agreement was $0.965(\mathrm{p}<0.001)$, and the sensitivity and specificity of the endoscopic criteria in predicting p-GR were 0.362 and 0.963 , respectively, for endoscopist B and 0.277 and 0.959 , respectively, for endoscopist C. The PPV and NPV of these criteria were 0.677 and 0.889 , respectively, for endoscopist B and 0.654 and 0.885 , respectively, for endoscopist $\mathrm{C}$. The false-positive and false-negative ratios were 0.323 and 0.111 , respectively, for endoscopist $B$ and 0.346 and 0.115 , respectively, for endoscopist C (Table 5).

\section{Discussion}

Several recent studies have reported on the feasibility of local excision of selected down-staged rectal cancers after preoperative CRT. In determining whether local excision is feasible in individual patients, proper evaluation of ypT after preoperative CRT is essential. Methods normally used for evaluation of ypT include digital rectal examination (DRE), imaging modalities (e.g., magnetic resonance imaging [MRI], ultrasound), and endoscopy. DRE is a simple and effective method of evaluating distal rectal lesions. Although very effective in detection of primary tumors, it has been found to underestimate the extent of rectal cancer response after preoperative CRT, suggesting that ypT should not be evaluated solely by DRE $[15,16]$. Ultrasound and MRI are also commonly used for preoperative evaluation of tumor stage in rectal cancer patients; however, evaluation by ultrasound was reported to be less accurate in assessing rectal tumor response after preoperative CRT [17], and MRI could not pre- dict pathological complete response after preoperative CRT [12]. Serum concentration of chorioembryonic antigen (CEA) and re-biopsy may be useful for predicting pathologic CR after preoperative CRT; however, evidence is still lacking $[12,13]$.

Currently, endoscopy is the only method to enable direct evaluation of rectal cancers after CRT, suggesting that it may be more effective for evaluation of ypT than other methods $[12,14]$. However, despite its efficacy, endoscopic descriptions remain subjective and non-standardized. Thus, there is a need for standardized criteria for endoscopic evaluation of ypT. In this study, the endoscopic findings of post-CRT rectal cancer were categorized according to seven categories-scarring, telangiectasia, erythema, nodule, ulcer, stricture, and remnant tumor-and these categories were used in classifying patients according to two response groups (e-GR and e-MR). Agreement between e-GR and p-GR was also analyzed. The kappa statistic of our endoscopic criteria was excellent (0.965), indicating that these criteria can be easily applied. We also found that this classification system has very high specificity (0.963) and NPV (0.885), but relatively low sensitivity (0.362) and PPV (0.654), suggesting that these endoscopic criteria can strongly predict $\mathrm{p}-\mathrm{MR}$ cases, but are less able to predict $\mathrm{p}-\mathrm{GR}$. Thus, these criteria can be helpful in selection of candidates for local excision.

Residual cancer cells in rectal cancer specimens after chemoradiation have been reported to be preferentially located close to the invasive front, not the mucosal surface [18], suggesting that endoscopic findings are not predictive of ypT0. Therefore, our classification system had high specificity, not sensitivity. In addition, some cases showed endoscopic findings not matching the pathologic response (Fig. 2). Development of a more accurate prediction system, with higher sensitivity, requires additional studies that include other methods of evaluation, including MRI, ultrasound, CEA, and / or clinical factors.

This study had several limitations. The study was retro- 

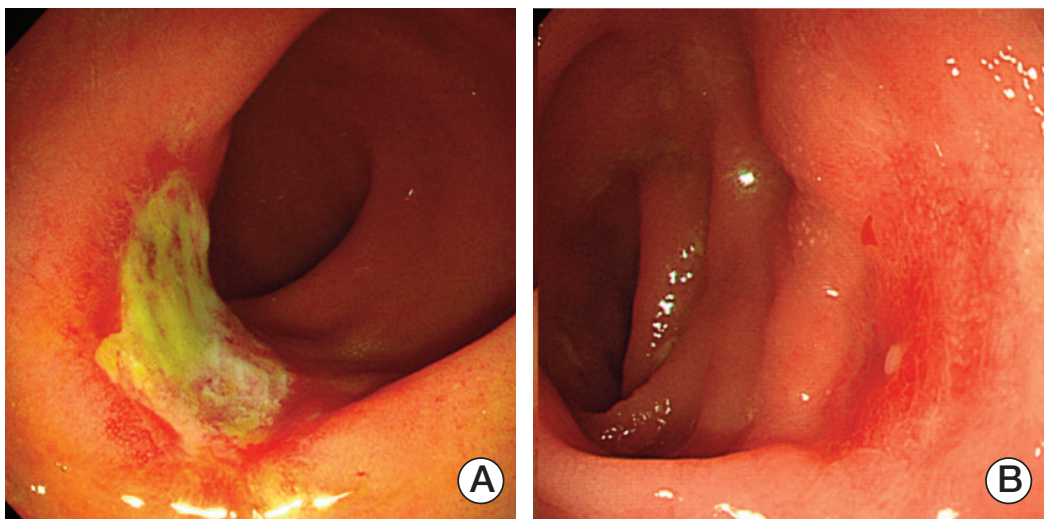

Fig. 2. Cases showing endoscopic findings not matching the pathological response. (A) Pathological good response (ypT0) showing endoscopic minimal or no response (ulcer). (B) Pathological poor response (ypT3) showing endoscopic good response (erythema).

spective in design and used recorded endoscopic pictures, some of which were limited. In addition, the number of patients who showed clinical or p-GRs may have been insufficient for data analysis. Conduct of large prospective studies will be required in order to validate our novel endoscopic criteria. Another limitation is that lymph node involvement of tumors was not considered in this study. The contraindication for local excision includes rectal cancers with lymph node metastasis. Therefore, evaluation of the lymph node status using imaging modalities is necessary prior to application of these criteria in clinical practice.

\section{Conclusion}

In conclusion, this study describes simple and objective endoscopic criteria for evaluation of the ypT of rectal cancer following preoperative CRT. These endoscopic criteria may be narrowly used to determine the suitability of patients for local excision, with or without additional radical surgery, following down-staging after preoperative CRT, since patients who do not show a pathologic good response could be ruled out.

\section{Conflicts of Interest}

Conflict of interest relevant to this article was not reported.

\section{Acknowledgments}

This work was supported by National Cancer Center Grants (NCC-1510150 and NCC-1510160-1).

\section{References}

1. Sauer R, Becker H, Hohenberger W, Rodel C, Wittekind C, Fietkau $\mathrm{R}$, et al. Preoperative versus postoperative chemoradiotherapy for rectal cancer. N Engl J Med. 2004;351:1731-40.

2. Sanghera P, Wong DW, McConkey CC, Geh JI, Hartley A. Chemoradiotherapy for rectal cancer: an updated analysis of factors affecting pathological response. Clin Oncol (R Coll Radiol). 2008;20:176-83.

3. Ikematsu H, Yoda Y, Matsuda T, Yamaguchi Y, Hotta K,
Kobayashi N, et al. Long-term outcomes after resection for submucosal invasive colorectal cancers. Gastroenterology. 2013;144:551-9.

4. Suh JH, Han KS, Kim BC, Hong CW, Sohn DK, Chang HJ, et al. Predictors for lymph node metastasis in T1 colorectal cancer. Endoscopy. 2012;44:590-5.

5. Lezoche E, Guerrieri M, Paganini AM, Baldarelli M, De Sanctis A, Lezoche G. Long-term results in patients with T2-3 N0 dis- 
tal rectal cancer undergoing radiotherapy before transanal endoscopic microsurgery. Br J Surg. 2005;92:1546-52.

6. Kundel Y, Brenner R, Purim O, Peled N, Idelevich E, Fenig E, et al. Is local excision after complete pathological response to neoadjuvant chemoradiation for rectal cancer an acceptable treatment option? Dis Colon Rectum. 2010;53:1624-31.

7. Yeo SG, Kim DY, Kim TH, Kim SY, Chang HJ, Park JW, et al. Local excision following pre-operative chemoradiotherapyinduced downstaging for selected cT3 distal rectal cancer. Jpn J Clin Oncol. 2010;40:754-60.

8. Lezoche E, Baldarelli M, Lezoche G, Paganini AM, Gesuita R, Guerrieri M. Randomized clinical trial of endoluminal locoregional resection versus laparoscopic total mesorectal excision for T2 rectal cancer after neoadjuvant therapy. Br J Surg. 2012; 99:1211-8.

9. Issa N, Murninkas A, Powsner E, Dreznick Z. Long-term outcome of local excision after complete pathological response to neoadjuvant chemoradiation therapy for rectal cancer. World J Surg. 2012;36:2481-7.

10. Gagliardi G, Newton TR, Bailey HR. Local excision of rectal cancer followed by radical surgery because of poor prognostic features does not compromise the long term oncologic outcome. Colorectal Dis. 2013;15:e659-64.

11. Pucciarelli S, De Paoli A, Guerrieri M, La Torre G, Maretto I, De Marchi F, et al. Local excision after preoperative chemoradiotherapy for rectal cancer: results of a multicenter phase II clinical trial. Dis Colon Rectum. 2013;56:1349-56.

12. Kuo LJ, Chiou JF, Tai CJ, Chang CC, Kung CH, Lin SE, et al. Can we predict pathologic complete response before surgery for locally advanced rectal cancer treated with preoperative chemoradiation therapy? Int J Colorectal Dis. 2012;27:613-21.

13. Habr-Gama A, Perez RO, Wynn G, Marks J, Kessler H, GamaRodrigues J. Complete clinical response after neoadjuvant chemoradiation therapy for distal rectal cancer: characterization of clinical and endoscopic findings for standardization. Dis Colon Rectum. 2010;53:1692-8.

14. Suzuki T, Sadahiro S, Tanaka A, Okada K, Okamura H, Machida T. Prediction of histologic regression on the basis of colonoscopic findings in patients with locally advanced middle and lower rectal cancer who receive preoperative chemoradiotherapy. Tokai J Exp Clin Med. 2011;36:100-5.

15. Hiotis SP, Weber SM, Cohen AM, Minsky BD, Paty PB, Guillem JG, et al. Assessing the predictive value of clinical complete response to neoadjuvant therapy for rectal cancer: an analysis of 488 patients. J Am Coll Surg. 2002;194:131-5.

16. Guillem JG, Chessin DB, Shia J, Moore HG, Mazumdar M, Bernard B, et al. Clinical examination following preoperative chemoradiation for rectal cancer is not a reliable surrogate end point. J Clin Oncol. 2005;23:3475-9.

17. Pastor C, Subtil JC, Sola J, Baixauli J, Beorlegui C, Arbea L, et al. Accuracy of endoscopic ultrasound to assess tumor response after neoadjuvant treatment in rectal cancer: can we trust the findings? Dis Colon Rectum. 2011;54:1141-6.

18. Duldulao MP, Lee W, Streja L, Chu P, Li W, Chen Z, et al. Distribution of residual cancer cells in the bowel wall after neoadjuvant chemoradiation in patients with rectal cancer. Dis Colon Rectum. 2013;56:142-9. 